\title{
CLASSIFICATION OF SIMPLICIAL TRIANGULATIONS OF TOPOLOGICAL MANIFOLDS
}

\author{
BY DAVID E. GALEWSKI ${ }^{1}$ AND RONALD J. STERN ${ }^{2}$
}

Communicated by S. Eilenberg, April 8, 1976

In this note we announce theorems which classify simplicial (not necessarily combinatorial) triangulations of a given topological $n$-manifold $M, n \geqslant 7$ ( $\geqslant 6$ if $\partial M=\varnothing)$, in terms of homotopy classes of lifts of the classifying map $\tau: M \longrightarrow$ $B T O P$ for the stable topological tangent bundle of $M$ to a classifying space $B T R I_{n}$ which we introduce below. The (homotopic) fiber of the natural map $j: B T R I_{n} \rightarrow B T O P$ is described in terms of certain groups of $P L$ homology 3 spheres. We also give necessary and sufficient conditions for a closed topological $n$-manifold $M, n \geqslant 6$, to possess a simplicial triangulation.

The proofs of these results incorporate recent geometric results of F. Ancel and J. Cannon [1], J. Cannon [2], R. D. Edwards [4], and D. Galewski and R. Stern [5].

In [8], R. Kirby and L. Siebenmann show that in each dimension greater than four there exist closed topological manifolds which admit no piecewise linear manifold structure and hence cannot be triangulated as a combinatorial manifold. Also, R. D. Edwards [3] has recently shown that the double suspension of the Mazer homology 3-sphere is homeomorphic to $S^{\mathbf{5}}$, thus showing that a simplicial triangulation of a topological manifold need not be combinatorial. But it is still unknown whether or not every topological manifold can be triangulated as a simplical complex.

Our classification theorems for simplicial triangulations on a given topological manifold take the following forms:

Let $B T O P$ denote the classifying space for stable topological block bundles.

THEOREM 1. There is a space BTRI $I_{n}$ and a natural map BTRI $I_{n} \rightarrow B T O P$ such that if $M$ is a topological $n$-manifold, $n \geqslant 7(\geqslant 6$ if $\partial M=\varnothing)$ and $\tau: M \longrightarrow$ BTOP classifies the stable topological tangent bundle of $M$, then there is a one-toone correspondence between the set of concordance classes of simplicial triangulations of $M$ and the set of vertical homotopy classes of lifts of $\tau$ to BTRI $I_{n}$.

\footnotetext{
AMS (MOS) subject classifications (1970). Primary 57C15.

${ }^{1}$ This research was supported in part by National Science Foundation grant GP29585-A4

${ }^{2}$ This research was supported in part by a Faculty Research Grant at the University of Utah and by National Science Foundation grant MCS76-06393.
} 
The obvious relative versions of Theorem 1 also hold true.

THEOREM 2. The fiber TOP/TRI $I_{n}$ of BTRI ${ }_{n} \rightarrow$ BTOP has only two nonzero homotopy groups, namely $\pi_{3}$ and $\pi_{4}$, and the following sequence is exact.

$$
0 \rightarrow \pi_{4} \rightarrow \operatorname{ker}\left(\alpha: \theta_{3}^{H} \rightarrow Z_{2}\right) \rightarrow \theta_{3}^{T R I_{n}} \rightarrow \pi_{3} \rightarrow 0 .
$$

Here $\theta_{3}^{H}$ denotes the group of $P L$ homology 3-spheres, modulo those which bound acyclic $P L$ 4-manifolds, under the operation of connected sum; $\alpha: \theta_{3}^{H} \rightarrow$ $Z_{2}$ is the Kervaire-Milnor-Rochlin map $\alpha\left(H^{3}\right)=I\left(H^{3}\right) / 8 \bmod 2$, where $I\left(H^{3}\right)$ is the index of a parallelizable $P L 4$-manifold that $H^{3}$ bounds; and $\theta_{3}^{T R I_{n}}$ is the group of $P L$ homology 3-spheres modulo those which bound acyclic homology 4-manifolds $W$ with $W \times R^{n-4}$ a topological manifold, under the operation of connected sum. Note that if $\Sigma^{n-3} H^{3}$ is homeomorphic to $S^{n}$, then $H^{3}$ represents the zero element of $\theta_{3}^{T R I_{n}}$.

THEOREM 3. (i) $\pi_{3}\left(T O P / T R I_{n}\right) \subseteq Z_{2}$,

(ii) $\pi_{3}\left(T O P / T R I_{n}\right)=0$ if and only if there exists a PL homology 3-sphere $H^{3}$ with $\alpha\left(H^{3}\right)=1$ and the $(n-3)$-suspension of $H^{3}, \Sigma^{n-3} H^{3}$, is homeomorphic to $S^{n}$.

(iii) $\pi_{4}\left(T O P / T R I_{n}\right)=0$ if and only if every PL homology 3-sphere $H^{3}$ with $\alpha\left(H^{3}\right)=0$ and which bounds an acyclic homology 4-manifold $W$ with $W \times R^{n-4}$ a topological manifold, bounds an acyclic PL 4-manifold.

THEOREM 4. There exists a PL homology 3-sphere $H^{3}$ such that

(i) $\alpha\left(H^{3}\right)=1$,

(ii) $H^{3} \# H^{3}$ bounds an acyclic PL 4-manifold, and

(iii) $\Sigma^{n-3} H^{3}$ is homeomorphic to $S^{n}$.

If and only if every closed topological $n$-manifold, $n \geqslant 6$, can be triangulated as a simplicial complex.

REMARK. For $M=5$ and $M^{n}$ oriented, Siebenmann [10] has shown under conditions (i) and (iii) that $M$ is simplicially triangulable. M. Scharlemann has pointed out that if $M^{5}$ is unoriented, then (i), (iii) and the fact that $H^{3} \# H^{3}$ bounds a contractible PL 4-manifold implies the result. For $6 \leqslant n \leqslant 8$, Theorem 4 was proven by M. Scharlemann [9], where in place of (ii) he has the orientability condition that the integral Bockstein of the Kirby-Siebenmann obstruction to putting a $P L$ structure on $M$ is zero. T. Matumoto has claimed a version of Theorem 4 under the stronger hypothesis that (iii) be replaced by the condition that $\Sigma^{n-4} H^{3}$ is homeomorphic to $S^{n-1}$.

We also investigate the question of whether a given topological $n$-manifold, $n \geqslant 6$, can be triangulated as a simplicial homotopy manifold. For example;

Proposition 5. Suppose that every PL homotopy 3-sphere bounds a cuntractible PL 4-manifold. Then there is a one-to-one correspondence between the set of concordance classes of simplicial homotopy manifold triangulations of 
a topological $n$-manifold $M, n \geqslant 6$, and concordance classes of PL manifold structures on $M$.

Proposition 6. Suppose there exists a bad counterexample to the 3 dimensional Poincaré conjecture; namely suppose there exists a PL homotopy 3sphere $H^{3}$, with

(i) $\alpha\left(H^{3}\right)=1$, and

(ii) $H^{3} \# H^{3}$ bounds a contractible PL 4-manifold.

Then every topological $n$-manifold, $n \geqslant 6$, can be triangulated as a simplicial homotopy manifold.

Details of these and related results will appear in [6] and [7].

\section{REFERENCES}

1. F. Ancel and J. W. Cannon, Any embedding of $S^{n-1}$ in $S^{n}(n \geqslant 5)$ can be approximated by locally flat embeddings, Notices Amer. Math. Soc. 23 (1976), p. A-308. Abstract \#732-G2.

2. J. Cannon, Taming codimension one generalized manifolds (preprint).

3. R. D. Edwards, The double suspension of a certain homology 3-sphere is $S^{5}$, Notices Amer. Math. Soc. 22 (1975), p. A-334. Abstract \#75T-G33.

4. The double suspension of PL homology n-spheres, Proc. Topology Conf. (Georgia, 1975).

5. D. Galewski and R. Stern. The relationship between homology and topological manifolds via homology transversality (preprint). print).

6. Classification of simplicial triangulations of topological manifolds (preprint).

7. - Surgery on compact simplicially triangulated topological manifolds (pre-

8. R. C. Kirby and L. C. Siebenmann, On the triangulation of manifolds and the Hauptvermutung, Bull. Amer. Math. Soc. 75 (1969), 742-749. MR 39 \#3500.

9. M. Scharlemann, Simplicial triangulations of non-combinatorial manifolds of dimen. sion less than nine, Inst. for Advanced Study, Princeton, N. J. (preprint).

10. L. C. Siebenmann, Are nontriangulable manifolds triangulable? Topology of Manifolds (Proc. Inst., Univ. of Georgia, Athens, Ga. 1969), (J. Cantrell and C. H. Edwards, Editors), Markham, Chicago, 1970, pp. 77-84. MR 42 \#6837.

DEPARTMENT OF MATHEMATICS, UNIVERSITY OF GEORGIA, ATHENS, GEORGIA 30601

UTAH 84112

DEPARTMENT OF MATHEMATICS, UNIVERSITY OF UTAH, SALT LAKE CITY, 